\title{
Retinoid X Receptor Agonist IRX4204
}

National Cancer Institute

\section{Source}

National Cancer Institute. Retinoid X Receptor Agonist IRX4204. NCI Thesaurus. Code C140551.

A second generation rexinoid and agonist of retinoid X receptor (RXR), with potential antineoplastic, neuroprotective, immunoregulatory, and disease-modifying activities. Upon administration, IRX4204 specifically binds to and activates RXRs, thereby activating RXR-mediated signaling pathways and inducing changes in gene expression that lead to cell differentiation. In responsive cancer cells, IRX4204 induces cell differentiation, decreases cell proliferation, and induces apoptosis, which leads to cancer regression. IRX4204 enhances the differentiation of CD4-positive T lymphocytes into inducible regulatory T cells (iT reg) and suppresses the development of inflammatory T helper 17 (Th17) cells. In addition, IRX4204 is able to cross the blood-brain barrier (BBB) and induces the differentiation of oligodendrocyte precursor cells (OPCs) into oligodendrocytes, promotes repair of myelin, and enhances the survival of dopaminergic neurons. 